\title{
A series of in vitro and human studies of a novel lip cream formulation for protecting against environmental triggers of recurrent herpes labialis
}

This article was published in the following Dove Medical Press journal:

Clinical, Cosmetic and Investigational Dermatology

\author{
Christoph F Gfeller' \\ Rita Wanser ${ }^{2}$ \\ Harish Mahalingam² \\ David J Moore ${ }^{3}$ \\ Xuying Wang ${ }^{4}$ \\ Connie B Lin ${ }^{4}$ \\ Gilbert Shanga ${ }^{5}$ \\ Gary Grove ${ }^{6}$ \\ Anthony $\vee$ Rawlings ${ }^{7}$ \\ 'GlaxoSmithKline Consumer \\ Healthcare, Medical Affairs Skin \\ Health, Weybridge, Surrey, UK; \\ ${ }^{2}$ GlaxoSmithKline Consumer \\ Healthcare, Medical Affairs \\ Skin Health, Warren, NJ, USA \\ ${ }^{3}$ GlaxoSmithKline Consumer \\ Healthcare, R\&D Innovation Skin \\ Health, Weybridge, Surrey, UK; \\ ${ }^{4}$ GlaxoSmithKline Consumer \\ Healthcare, R\&D Innovation Skin \\ Health, Collegeville, PA, USA; ${ }^{5}$ GSK \\ Consumer Healthcare, Biostatistics, \\ Warren, NJ, USA; ' ${ }^{6}$ cyberDERM, Inc., \\ Broomall, PA, USA; ${ }^{7}$ AVR Consulting, \\ Ltd., Cheshire, UK
}

Correspondence: Harish Mahalingam GlaxoSmithKline Consumer Healthcare, Medical Affairs Skin Health, 184 Liberty Corner Rd, Warren, NJ 07059, USA

Tel + I 9082934000

Email harish.x.mahalingam@gsk.com
Purpose: These studies describe the testing of a novel, daily-use lip cream designed for individuals with lips prone to recurrent herpes labialis (RHL) that protects against environmental triggers. Subjects and methods: In vitro occlusive and in vitro and in vivo photoprotection analyses, a characterization of normal vs dry lips, and a randomized, evaluator-blinded, clinical trial that assessed the lip cream in healthy subjects with dry lips were conducted. In the clinical trial, subjects applied the lip cream or were untreated and evaluated using transepidermal water loss (TEWL), corneometry, visual assessments of lip dryness, expert photographic evaluations, and subject-rated outcomes.

Results: The lip cream's in vitro water vapor transmission rate $\left(84.1 \mathrm{~g} /\left(\mathrm{m}^{2} \mathrm{~h}\right)\right)$ indicated moderate occlusivity. The lip cream, but not placebo or control (water), reduced ultraviolet A (UVA)- and UVB-induced DNA damage, and tumor necrosis factor- $\alpha$ (EpiDerm ${ }^{\mathrm{FT}}$ ) and prostaglandin $\mathrm{E}_{2}$ release $\left(\right.$ EpiDerm $^{\mathrm{FT}}$ and EpiGingival $\left.{ }^{\mathrm{TM}}\right)$. The lip cream's in vivo sun protection factor (SPF) was 12.2 (lower confidence limit, 11.3) and SPF/UVA protection factor ratio was 0.9. The characterization of dry vs normal lips identified differences in moisturization. In the clinical trial, the lip cream significantly decreased TEWL (difference: -7.19 [95\% CI: -11.41 , -2.98]; $P<0.01$ ), increased corneometry (difference: 4.62 [95\% CI: $1.05,8.19$ ]; $P<0.05$ ), and reduced visual dryness (difference: -1.48 [95\% CI: $2.24,-0.71]$; $P<0.001$ ) compared to untreated subjects. Significant benefits were also observed on expert photographic assessments of scaling (difference: -0.89 [95\% CI: $-1.75,-0.03]$; $P<0.05$ ), cupping (difference: -1.50 [95\% CI: $-2.30,-0.70] ; P<0.001$ ), and healthy appearance (difference: -1.44 [95\% CI: $-2.29,-0.58]$; $P<0.01)$; differences in overall healthy appearance were not significant $(P=0.51)$. Subject-rated assessments indicated improvements in cracking, dryness, and flaking in the lip cream group but worsening in untreated subjects.

Conclusion: These studies indicate that this novel, daily-use lip cream protects against UV radiation, drying, and chapping, which are established environmental RHL triggers.

Keywords: herpes simplex virus, barrier function, lip care, UV radiation

\section{Introduction}

Lip skin, more precisely the vermilion lip, has characteristics and properties that differentiate it from the skin in other areas of the body, suggesting that the lips require specialized protection and care. Specifically, lip skin lacks sweat glands and sebaceous glands are found in only $50 \%$ of the postadolescent population. ${ }^{1,2}$ Furthermore, lip skin is also only lightly keratinized and contains little melanin. ${ }^{1-3}$ Given the role these skin components play in providing endogenous photoprotection from ultraviolet (UV) radiation, the lips are predisposed to the negative effects of the sun and UV damage. ${ }^{4}$ 
Lip skin is also characterized by a thinner stratum corneum (SC) that contains less ceramides and those present have a shorter chain length than skin in most other areas of the body. ${ }^{5,6}$ Moreover, it is known generally that facial SC contains less filaggrin-derived natural moisturizing factor than other body sites. ${ }^{7}$ As a result, higher levels of transepidermal water loss (TEWL) and lower electrical measures of hydration have been observed with lip skin compared to skin in many other areas of the body. ${ }^{1,6}$

These combined distinguishing characteristics of lip skin, in addition to the lips' high exposure to environmental conditions, are directly related to the tendency for lip skin to become dry, rough, chapped, and susceptible to damage from UV radiation and other environmental factors. ${ }^{1-3,8}$

Recurrent herpes labialis (RHL) is caused by a herpes simplex virus (HSV) infection, most commonly HSV type 1 (HSV-1) and the WHO has estimated that as of 2012, 67\% of the world's population is infected with HSV-1. ${ }^{9}$ Approximately $14 \%-42 \%$ of HSV-1 seropositive individuals develop RHL (cold sores) at least once in their lives. ${ }^{10}$ Although there is the potential for spontaneous recurrences, RHL is thought to often be triggered by endogenous or exogenous factors, including stress, fatigue, immunosuppression, local tissue trauma, exposure to heat, cold, or UV radiation, or a disruption of the skin barrier, including dry and chapped lips. ${ }^{2,11-18}$ Characterization of a herpes simplex labialis population has shown that winter and summer have the highest rates of cold sore outbreaks with extreme weather conditions or temperatures being among the top triggers. ${ }^{19}$ UV sunlamps have been used to experimentally induce lesions in individuals with RHL-prone lips with the goal of developing a methodology for use in subsequent clinical trials of RHL treatments, clearly demonstrating the role of UV radiation in the reactivation of $H S V \cdot{ }^{13,14,20}$ As is well known, UV exposure also causes inflammation, which can induce proinflammatory mediators, such as tumor necrosis factor- $\alpha$ (TNF- $\alpha$ ), IL, and prostaglandin $\mathrm{E}_{2}\left(\mathrm{PGE}_{2}\right){ }^{21,22} \mathrm{PGE}_{2}$, in particular, is involved in a variety of immune responses and further associated with the development of RHL, although the mechanisms through which this effect occurs have not been clearly established. ${ }^{14,23-26}$

The best prophylaxis likely to be beneficial for herpes labialis has been reported to be oral antivirals and topical sunscreens. ${ }^{27,28}$ The benefit of the latter has been questioned when comparing experimental testing vs skiers exposed to natural conditions. ${ }^{29-31}$ However, recent research has provided evidence for the benefit of sunscreens in preventing RHL from a randomized crossover study of a lipstick with and without sunscreen protection while subjects performed their normal daily activities including trips to the beach. ${ }^{32}$ Equally, a zinc oxide or sulfate "indifferent" cream and gel have also been shown to have a small favorable effect on the duration of cold sore symptoms. ${ }^{33}$ The authors focused on the effects of zinc but moisturization may also be a mechanism involved as occlusion has also been proposed as a treatment. Moreover, a novel protective barrier gel containing oxygenated glycerol triesters has recently been developed for RHL. ${ }^{34}$

Protecting RHL-prone lips from UV radiation exposure and dehydration could potentially reduce the risk of future RHL episodes. ${ }^{19,35}$ However, in contrast to the numerous studies that have been conducted with topical products to treat dry body skin or protect face and body skin from UV radiation, the lips have not been as widely studied. However, even in the small number of studies conducted to treat dry lip skin with topical products, efficacy was demonstrated generally based on changes from the baseline dry lip condition. ${ }^{36-38}$

Due to the unique needs of lip skin, in particular with regard to preventing RHL, a novel lip cream formulation was developed containing UV filters and moisturizers that protect and repair lip skin. This formulation was developed with a novel and proprietary Micro Repair technology that enhances barrier function through including hydrogenated phospholipids, behenyl alcohol, and other plant-derived lipids that together form a similar lamellar structure to that found naturally in the SC that do not disrupt the SC lipids as some other emollients are known to do (Table 1). ${ }^{39-41}$ Mineral oil-derived saturated hydrocarbons were also excluded; their incorporation in lip care products has been requested to be minimized by Cosmetics Europe. ${ }^{42}$ This formulation also contains other important ingredients that help to maintain healthy lips, including humectants (ie, glycerin, at levels that meet the skin protectant monograph, and glucose, which are also keratinocyte differentiation enhancers and exert antiinflammatory action) and UVA and UVB filters to reduce lip skin inflammation. ${ }^{43-48}$ This report describes a series of in vitro and in vivo studies that were conducted to develop, optimize, and clinically test a daily-use lip cream formulation to protect against some of the known triggers of RHL.

\section{Subjects and methods}

\section{In vitro evaluation of occlusivity}

A uniform amount of the lip cream formulation or $100 \%$ petrolatum (as a positive control) was applied to a weighted portion of an in vitro skin model (Vitro-Skin, IMS Inc., Portland, ME, USA). An untreated control sample was prepared in the same manner. ${ }^{39}$ The treated and control samples were 
Table I Product composition and usage instructions

\begin{tabular}{|l|l|}
\hline Ingredients & $\begin{array}{l}\text { Aqua, glycerin, glucose, diethylhexyl syringylidene malonate, butyrosperum parkii butter, olus oil, ethylhexyl salicylate, oryza } \\
\text { sativa cera, butyl methoxydibenzoylmethane, behenyl alcohol, caprylic/capric triglyceride, oryza sativa bran oil, capryloyl } \\
\text { glycine, hydrogenated lecithin, pentylene glycol, parfum, caprylyl glycol, dehydroxanthan gum, sodium hydroxide, squalane, } \\
\text { tocopherol, polyvinylpyrrolidone/eicosene copolymer, acrylates/CI0-30 alkyl acrylate crosspolymer, sodium carbomer, trisodium } \\
\text { ethyldiamine disuccinate, palmitamide MEA, ascorbyl palmitate, ceramide 3, phytosphingosine }\end{array}$ \\
\hline Use & $\begin{array}{l}\text { Topically apply a sufficient amount of product to cover each lip } 6 \text { times daily (upon awakening, after breakfast, after lunch, mid/ } \\
\text { late afternoon, after dinner, and prior to bedtime) } \\
\text { Each application is estimated to be } 0.0285 \mathrm{~g}, \text { for a total of } 0.17 \mathrm{~g} / \text { day } \\
\text { Do NOT apply on the day of scheduled study visits until after all visit procedures are completed }\end{array}$ \\
\hline
\end{tabular}

placed over water for 3 hours and weighed periodically. The water vapor transmission rate (WVTR) was then determined for each sample, represented as $\mathrm{g} /\left(\mathrm{m}^{2} \mathrm{~h}\right)$. Only descriptive statistics were calculated and are reported for this outcome.

\section{In vitro evaluation of photoprotection}

Three in vitro reconstructed human tissue models were used to determine the degree of photoprotection provided by the test lip cream. The EpiDerm and EpiDerm ${ }^{\mathrm{FT}}$ (MatTek; Ashland, MA, USA) models were used, which consist of normal, human-derived epidermal keratinocytes with and without dermal fibroblasts, respectively. These models have been well established as research tools to evaluate the photoprotective effect of sunscreens in vitro. The EpiGingival ${ }^{\mathrm{TM}}$ (MatTek) model was also used, which consists of normal human oral keratinocytes that are differentiated into tissues with a cornified, gingival phenotype that is considered to be an appropriate representation of lip skin. ${ }^{49-52}$ The tissue models were topically treated with distilled water (control), the test lip cream formulation without UV filters (placebo), or the test lip cream formulation with UV filters. One hour after topical treatment, the tissues were exposed to either UVA $(30,50$, or $\left.70 \mathrm{~J} / \mathrm{cm}^{2}\right)$ or UVB $\left(150 \mathrm{~mJ} / \mathrm{cm}^{2}\right)$ radiation. EpiDerm was used to evaluate UVB-induced damage, EpiDerm ${ }^{\mathrm{FT}}$ was used to evaluate UVA-induced damage, and EpiGingival was used to evaluate both UVA- and UVB-induced damage.

The irradiated tissue models were evaluated for DNA damage, apoptosis, and various inflammatory mediators. Immunohistochemical analysis of DNA damage was performed by measuring cyclobutane pyrimidine dimers (CPDs), a marker for UVB damage, and 8-hydroxy-2'deoxyguanosine (8OHdG), a marker for UVA damage. Immunohistochemical analysis of apoptosis was performed by measuring cleaved caspase-3 (CC3). Concentrations of IL-6, IL-8, TNF- $\alpha$, and PGE $_{2}$ released from the tissues were also measured. A Student's $t$-test was used for comparisons between test formulations.

\section{In vivo sun protection factor (SPF) study}

The SPF of the lip cream formulation was determined according to International Organization for Standardization (ISO 24444) standards and the US Food and Drug Administration (FDA) requirements. ${ }^{53-55}$ Before initiating the trial, the investigator obtained written and dated full approval from the Freiburg Ethics Commission International for the protocol and any amendments, and collected the subjects' signed informed consent forms.

Eligible subjects were males or females between 18 and 70 years of age with Fitzpatrick skin phototypes I-III and three test areas measuring $5 \mathrm{~cm} \times 8 \mathrm{~cm}$ marked on their backs between the waist and shoulders were used. The untreated test area was irradiated using a sun simulator (300W Multiport, SOLAR Light Company, Glenside, PA, USA) and was evaluated within 16-24 hours after irradiation to determine the minimum erythemal dose (MED) for each subject. One week later, the negative control (untreated area) was irradiated to estimate the MED of unprotected skin. Also, a control sunscreen product (SPF Standard P2, with Padimate $\mathrm{O}$ and Oxybenzone) with an SPF rating of 16 and the lip cream formulation were applied to the two remaining test areas. The test areas were irradiated 15-30 minutes after application and evaluated 16-24 hours later at day 3 to determine MED and calculate the SPF. The SPF value of the control product was required to be within the expected range (ie, $16 \% \pm 17 \%$ ). Adverse events (AEs) were recorded for all irradiated subjects.

\section{In vitro UVA protection factor (UVAPF) study}

Spectroradiometry was performed to determine the in vitro UVAPF in accordance with ISO 24443 guidelines. ${ }^{54}$ The critical wavelength and ratio of the in vivo SPF to the in vitro UVAPF were calculated. The Commission Recommendation of the European Union requires that these values be above 370 $\mathrm{nm}$ and have a ratio $<3$, respectively, for cosmetic products that claim UVA protection. ${ }^{56}$ 


\section{Characterization of dry and normal lips}

This study was conducted during December 3-18, 2013, in Philadelphia, USA. All subjects provided written informed consent, and all study materials received institutional review board approval prior to study initiation.

Fifty female subjects, aged $18-45$ years, who had not used lip products for 2 days, were recruited with self-perceived and expertly assessed normal (grade 0 on a 10-point visual dryness assessment scale) or self-perceived and expertly assessed dry lips (grade 3 or 4). Objective evaluations included TEWL (cyberDERM RG1 Evaporimeter System, cyberDERM Inc.), capacitance (Corneometer ${ }^{\circledR}$ CM 825, Courage + Khazaka electronic GmbH, Cologne, Germany), conductance (DermaLab ${ }^{\circledR}$ Moisture Meter [DMM], Cortex Technology, Hadsund, Denmark), and determination of protein content obtained from tape strippings (4 D-SQUAME ${ }^{\circledR}$, CuDerm, Dallas, TX, USA) using a SquameScan ${ }^{\circledR} .^{57}$

The primary analysis involved calculating descriptive statistics for TEWL, corneometry, and DMM measurements (ie, three TEWL measurements together with five corneometry and DMM measurements). Two sample $t$-tests were performed using a mixed model at a 5\% significance level with cohort as a fixed effect; 95\% CIs were calculated for comparisons of within- and between-cohort means.

\section{Efficacy trial: improving dry lips}

Following on from the characterization study, this randomized, evaluator-blinded, parallel-group, single-center study assessed the efficacy of the test lip cream for improving dry lips compared with untreated control subjects during February 26 and March 14, 2014 in Philadelphia, USA.

Healthy females aged 18-55 years with dry lips of grade 4 , or 5-6 based on a 9-point visual dryness assessment scale who had not used products for 1-3 days were recruited. A photonumeric scale was developed to provide a visual representation of the rating for each range of values from this study (Figure 1) after which baseline lip photographs together with corneometry and TEWL measurements were taken. Subjects in the active treatment group applied the lip cream formulation 6 times per day for 1 week $(0.0285 \mathrm{~g} /$ application; total: $0.17 \mathrm{~g} /$ day), while subjects in the untreated group were restricted from applying any products to their lips for the duration of the study. Compliance, visual assessment of lip dryness, corneometry, TEWL, and photographs (standard and polarized) were evaluated on days 3 and 8 for both groups and on day 9 (24 hours post-treatment) for the treatment group. Subjects also rated the condition and appearance of their own lips together with the level of protection, strength, moisturization, and healthy appearance that the test lip cream provided using 5-point grading scales. Changes in lip appearance were then assessed by comparing the baseline photographs with those taken on day 8 . The photographs were rated on healthy appearance $(0=$ acceptable; $9=$ not acceptable), scaling $(0=$ none; $6=$ generalized lifting scale, severe [ $\geq 51 \%$ of the lip area]), and cupping ( $0=$ full, plump; $6=$ entire surface cupping) by presenting baseline and day 8 photographs for the same subject in a random order. The expert graders were then asked to provide their preference for a photograph and to rate the difference between them $(0=$ no detectable difference; $3=$ great difference).

Analysis of covariance was used to compare the lip cream formulation group with the untreated group for all measures (ie, visual dryness assessment and TEWL on days 3 and 8; corneometry on day 8). Ninety-five percent CIs were calculated for the least squares means and differences. Change from baseline values was used in the analysis as the response variable, and other factors in the model included treatment as a fixed effect and baseline values as a covariate. All tests were performed at a $5 \%$ significance level.

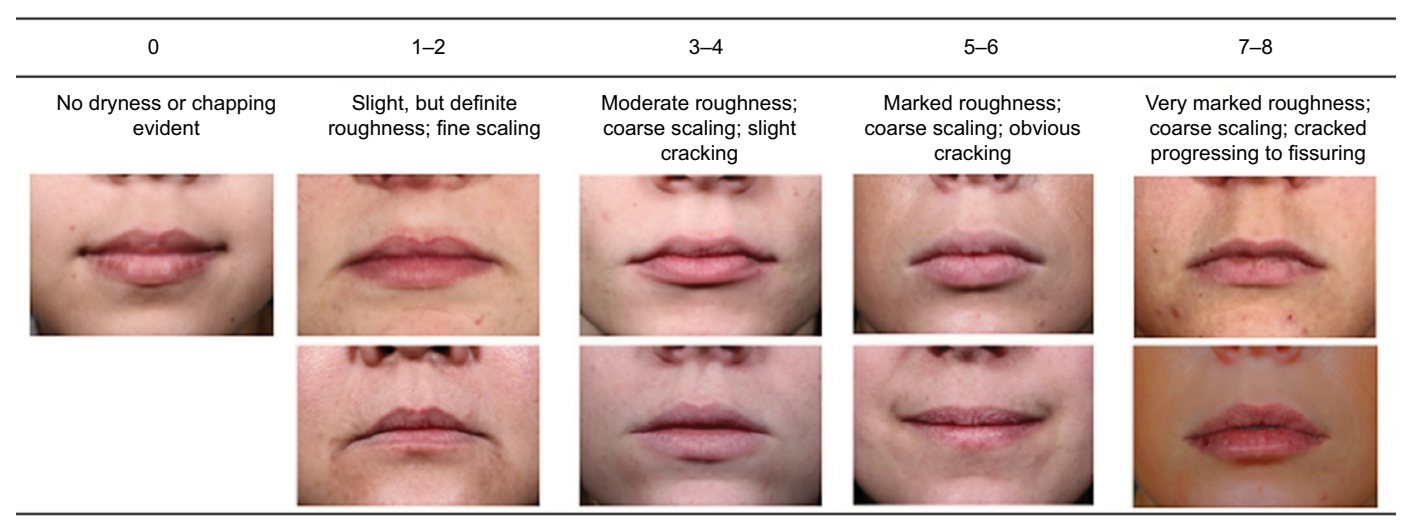

Figure I Photonumeric lip dryness grading scale. 


\section{Results}

\section{In vitro evaluation of occlusivity}

The test lip cream formulation was found to be moderately occlusive, with a WVTR of $84.1 \mathrm{~g} /\left(\mathrm{m}^{2} \mathrm{~h}\right)$, which was significantly different from the untreated control $(P<0.001)$ (Figure 2). As expected, the untreated control model had a high WVTR value. However, the $100 \%$ petrolatum was found to be highly occlusive $\left(0.73 \mathrm{~g} /\left(\mathrm{m}^{2} \mathrm{~h}\right)\right)$, with almost no transmission of water vapor.

\section{In vitro evaluations of photoprotective properties}

UVB-irradiated EpiDerm and EpiGingival tissues that were treated with the lip cream formulation showed little to no immunohistochemical staining of UVB-induced CPD and CC3. In contrast, a high level of CPD staining was observed in the control and placebo-treated samples. These results indicate that the lip cream formulation with UV filters protected against UVB-induced DNA damage and apoptosis (Figures 3 and S1).

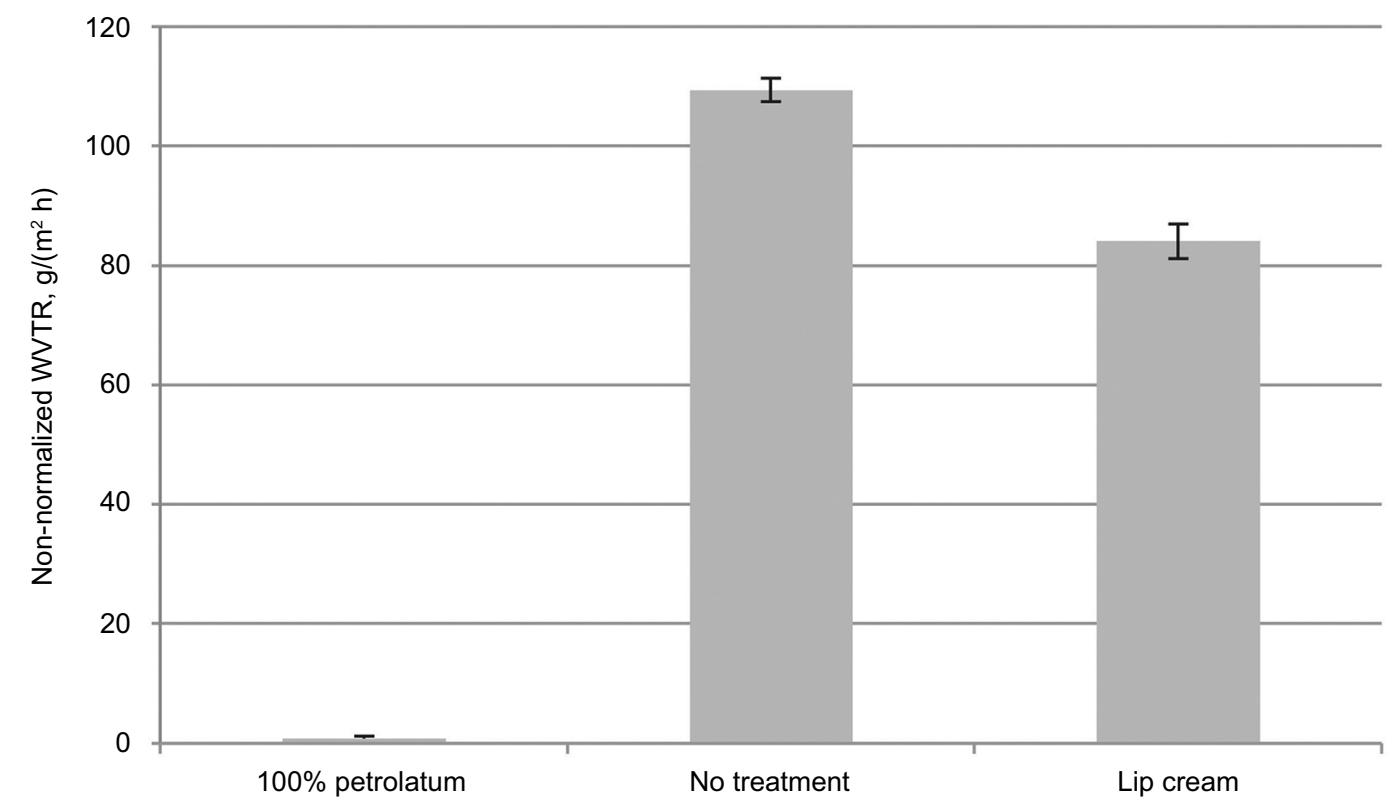

Figure 2 In vitro water vapor transmission rate of the lip cream formulation, 100\% petrolatum, and no treatment applied to a skin model. Abbreviation: WVTR, water vapor transmission rate.

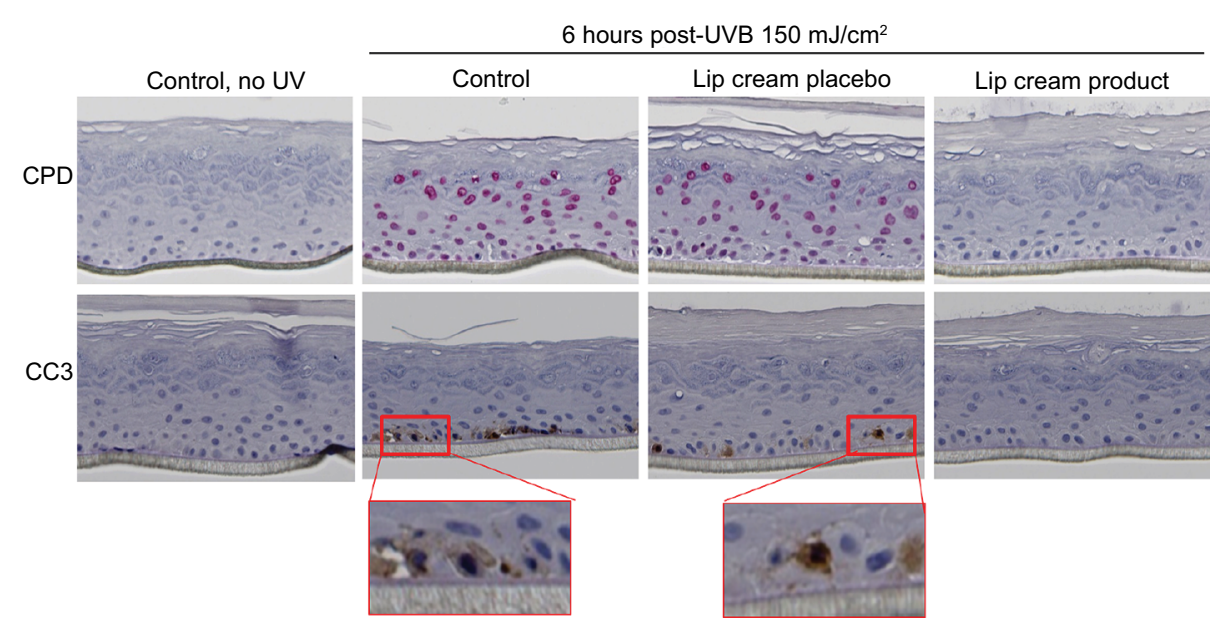

Figure 3 Lip cream with UV filters inhibited UVB-induced DNA damage (CPD, pink staining) and apoptosis (CC3, brown staining) in EpiDerm.

Notes: Lip cream with UV filters and without UV filters (placebo) were topically applied I hour prior to UVB irradiation (I50 mJ/cm²). Non-UVB treatment (untreated) was used as a negative control and baseline. Tissue samples were collected for IHC staining at 6 hours post-UVB ( $n=3$ ). The images at top panels were scanned at $40 \times$ using Nanozoomer (Hamamatsu) and shown at 100\%. The images in red boxes shown at bottom panels were manually magnified 3 times from the original images.

Abbreviations: CC3, cleaved caspase-3; CPD, cyclobutane pyrimidine dimers; IHC, immunohistochemical; UVB, ultraviolet B. 
UVB irradiation resulted in increases in the release of proinflammatory mediators IL- 8 , TNF- $\alpha$, and $\mathrm{PGE}_{2}$ from the control and placebo-treated EpiDerm samples. The test lip cream with UV filters significantly reduced the UVB-induced IL-6, IL-8, TNF- $\alpha$, and PGE $_{2}(P<0.05$ vs the placebo-treated samples; Figure 4A). Similar PGE2 inhibition results were obtained in the EpiGingival model (Figure 4B), although UVB radiation did not significantly induce IL-8 (data not shown). UVA irradiation $\left(30 \mathrm{~J} / \mathrm{cm}^{2}\right)$ strongly induced TNF- $\alpha$ and $\mathrm{PGE}_{2}$ release from the control and placebo-treated EpiDerm ${ }^{\mathrm{FT}}$ samples but not from the samples treated with the test lip cream. Samples treated with the lip cream with UV filters showed significantly lower induction of TNF- $\alpha$ compared to those treated with placebo $(P<0.05$; Figure $4 C)$. Similar results were seen in the EpiGingival model on $\mathrm{PGE}_{2}$ (Figure 4D). UVA irradiation $\left(70 \mathrm{~J} / \mathrm{cm}^{2}\right)$ led to dark brown staining of $8 \mathrm{OHdG}$ with atypical nuclei and strong staining of $\mathrm{CC} 3$ in the control and placebo-treated EpiDerm ${ }^{\mathrm{FT}}$ samples, demonstrating DNA damage and apoptosis. The test lip cream formulation reduced UVA-induced DNA damage and apoptosis. As shown in Figure 5A, B, the EpiDerm ${ }^{\mathrm{FT}}$ samples treated with the test lip cream had light brown 8OHdG staining with normal nuclei, no CC3 staining, and no tissue damage. Tissue damage, as evidenced by separation of the epidermis and dermis, was observed in the EpiDerm ${ }^{\mathrm{FT}}$ (Figure 5A, B) and EpiGingival samples (Figure S2) treated with placebo but not in tissues treated with the test lip cream. Based on these differences, the EpiGingival model appears to be more vulnerable to UVA irradiation than EpiDerm ${ }^{\mathrm{FT}}$. These in vitro data show that the test lip cream formulation that contains UV filters provides broad-spectrum protection from UV radiation, as demonstrated by reduced UV-induced proinflammatory mediators, DNA damage, and apoptosis.

\section{In vivo SPF study \\ Demographics}

Sixteen subjects were enrolled for the ISO 24444 analysis. Of these subjects, 12 were included in the per-protocol population, 10 were female, and the mean age was 48.7 years. Five subjects each had skin phototype I and II, and the remaining two subjects had skin phototype III. Thirteen subjects were enrolled for the FDA Final Rule analysis. Of these subjects,
A
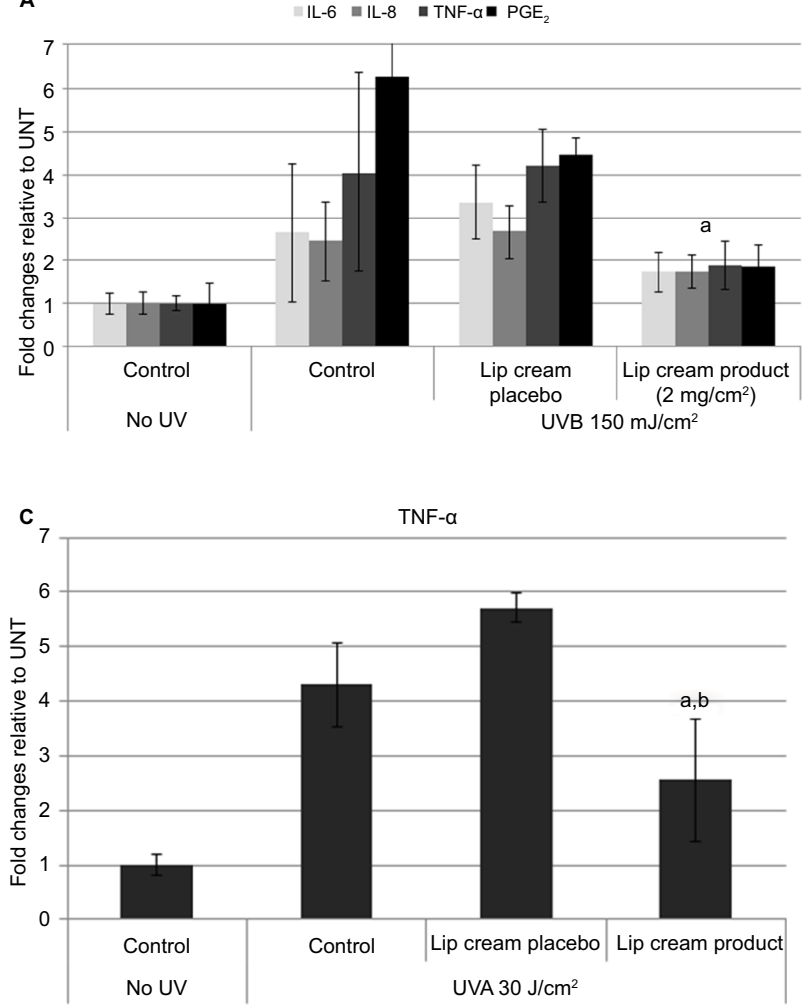

B

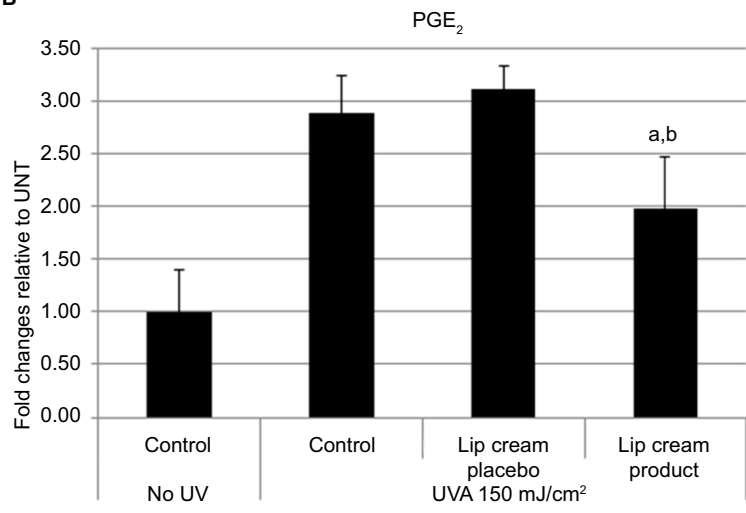

D

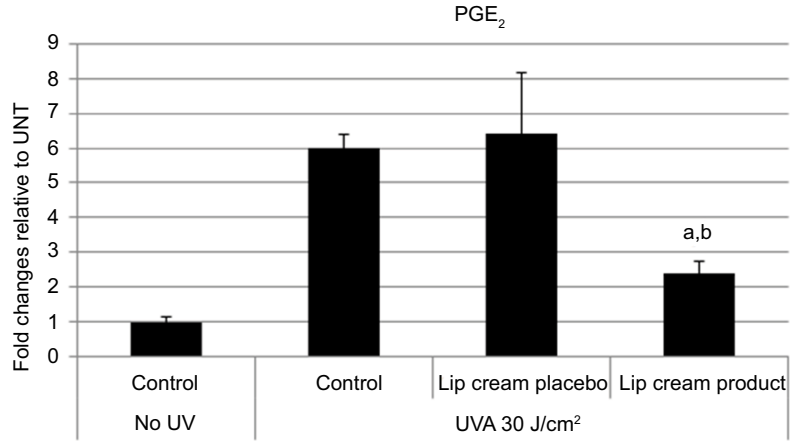

Figure 4 Lip cream with UV filters significantly reduced proinflammatory mediators induced by UVB.

Notes: Lip cream with and without UV filters (placebo) were topically applied I hour prior to UVB exposure $\left(150 \mathrm{~mJ} / \mathrm{cm}^{2}\right)$ in EpiDerm (A) and EpiGingival ${ }^{\top M}$ (B), or I hour prior to UVA exposure $\left(30 \mathrm{~J} / \mathrm{cm}^{2}\right)$ in EpiDerm ${ }^{F T}(\mathbf{C})$ and EpiGingival (D). Non-UV treatment (UNT) was used as a negative control and baseline $(\mathrm{n}=3)$. aP<0.05 for lip cream with UV filters vs UVA or UVB alone. ${ }^{b P}<0.05$ for lip cream with UV filters vs placebo.

Abbreviations: $\mathrm{PGE}_{2}$, prostaglandin $\mathrm{E}_{2}$; TNF- $\alpha$, tumor necrosis factor- $\alpha$; UNT, untreated; UVA, ultraviolet $A$; UVB, ultraviolet $B$. 
A

$8 O H d G, 6$ hours post-UVA $70 \mathrm{~J} / \mathrm{cm}^{2}$

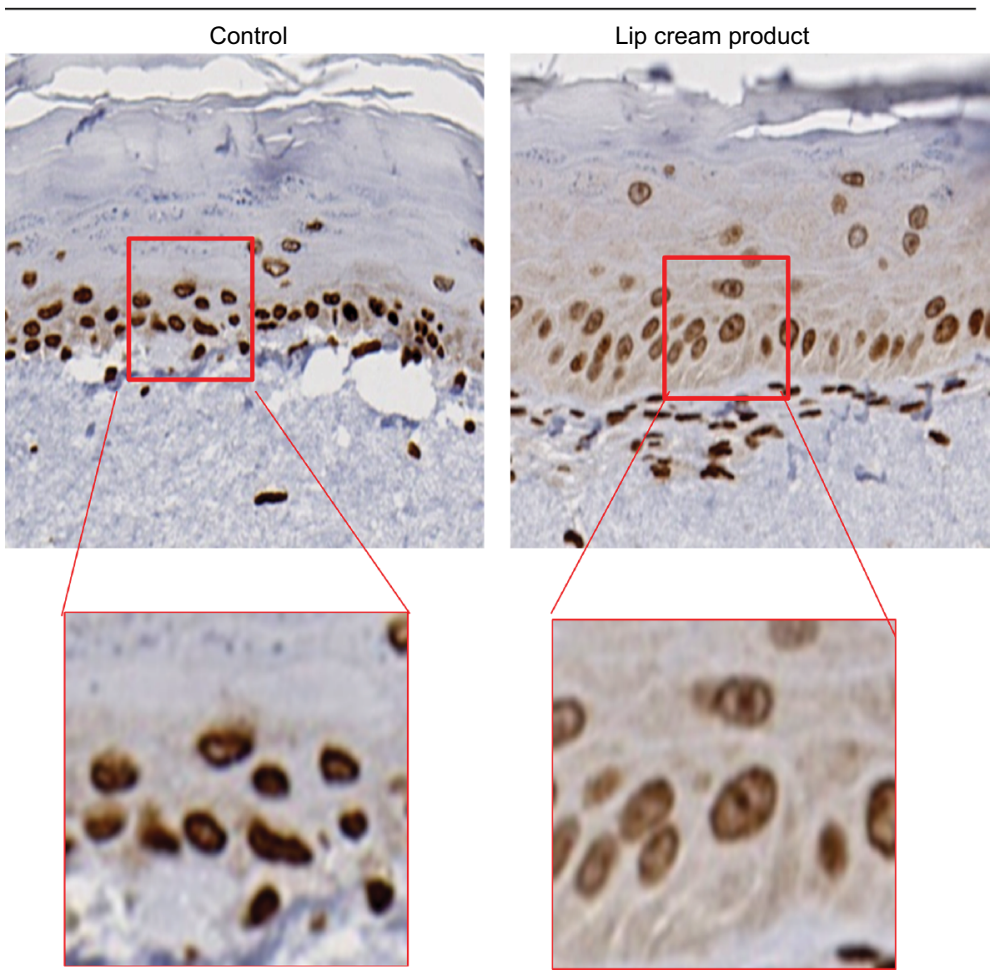

B CC3, 6 hours post-UVA $70 \mathrm{~J} / \mathrm{cm}^{2}$

Control Lip cream product
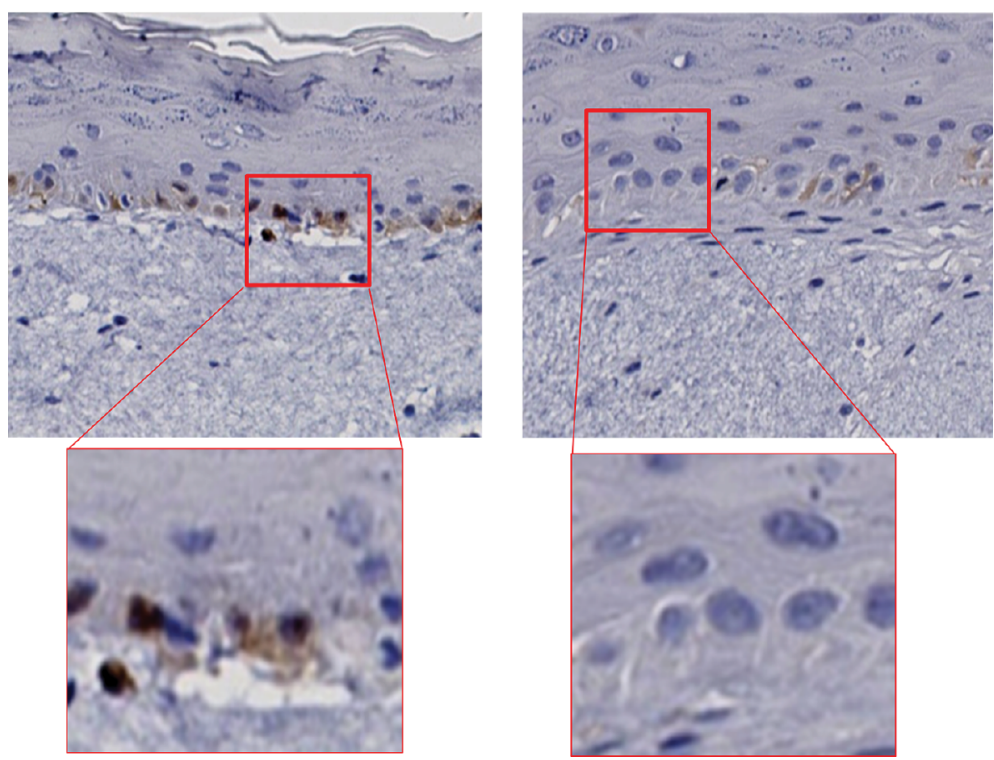

Figure 5 Lip cream with UV filters inhibited UVA-induced DNA damage (8OHdG, dark brown nuclear staining); (A) and apoptosis (CC3, brown staining,) (B) in EpiDerm ${ }^{F T}$ at 6 hours post-UVA $\left(70 \mathrm{~J} / \mathrm{cm}^{2}\right)$.

Notes: Lip cream placebo is the test formulation without the UV filters. Untreated samples were not applied with any test product $(n=3)$. (A and B) The images at top panels were scanned at 40x using Nanozoomer (Hamamatsu) and shown at 100\%. (A and B)The images in red boxes shown at bottom panels were manually magnified 3 times from the original images.

Abbreviations: 8OHdG, 8-hydroxy-2'-deoxyguanosine; CC3, cleaved caspase-3; UVA, ultraviolet A. 
ten were included in the per-protocol population. Nine subjects were female, and the mean age of the population was 50 years. Four subjects each had skin phototype I and II, and the remaining two subjects had skin phototype III.

\section{In vivo SPF}

For the ISO 24444 analysis, the SPF of the control sunscreen product was determined to be 13.8 with a $\mathrm{CI}$ of $\pm 17.0 \%$. The SPF of the test lip cream formulation was determined to be 12.1 ( $\mathrm{CI} \pm 14.5 \%$ ). For the FDA Final Rule analysis, the SPF of the control sunscreen product was 14 (lower confidence limit 12.7), and the SPF of the test lip cream was 12.2 (lower confidence limit 11.3). The observed SPF of the control sunscreen product was consistent with the labeling, confirming the accuracy of the study methods. According to the definition of the Commission Recommendation of the European Union, the labeled SPF of the lip cream is "low", and based on the FDA Final Rule definition, the labeled SPF of the test lip cream is 10 . No AEs were reported during the study.

\section{In vitro assessment of UVAPF}

In this in vitro analysis, the mean UVAPF of the lip cream was determined to be 10.8; therefore, the ratio of the in vivo SPF of 10 to the in vitro UVAPF was 0.9 . The mean critical wavelength of the lip cream was calculated to be $377.6 \mathrm{~nm}$. Therefore, these results meet the criteria of the Commission Recommendation of the European Union of an SPF/UVAPF ratio $<3$ for cosmetic products that claim to provide protection from UVA radiation and a mean critical wavelength of $>370 \mathrm{~nm}$ for products that claim to provide broad-spectrum protection from UV radiation. The SPF human data and the in vitro UVAPF data indicate that the test lip cream provides broad-spectrum protection from UV radiation.

\section{Characterization of dry and normal lips}

Forty-four female subjects were enrolled and evenly divided between the two groups ( $n=22$ per group). One subject was excluded because of a protocol violation, while the remaining 43 subjects completed the study. The mean age of the study population was 35.3 years (range, $18-45$ years).

There was no statistical difference in mean TEWL measurements between the subjects with dry lips and those with normal lips (Table 2). Subjects with normal lips had significantly higher mean values on the DMM compared to subjects with dry lips $(P<0.01)$. Similarly, subjects with normal lips had significantly higher mean corneometry measurements compared to those with dry lips $(P<0.01)$. These data show that DMM and corneometry measurements were
Table 2 Characteristics of normal lips and dry lips, measured by mean TEWL, DMM, and corneometer values

\begin{tabular}{|l|l|l|}
\hline $\begin{array}{l}\text { Measurement, } \\
\text { mean (SD) }\end{array}$ & $\begin{array}{l}\text { Subjects with } \\
\text { normal lips } \\
\text { (n=22) }\end{array}$ & $\begin{array}{l}\text { Subjects with } \\
\text { dry lips } \\
\text { (n=22) }\end{array}$ \\
\hline TEWL, g/(m ${ }^{2}$ h) & $45.6(12.6)$ & $46.4(11.8)$ \\
DMM, $\mu$ s & $106.1(60.9)$ & $66.8(23.7)$ \\
Corneometer, AU & $38.7(13.9)$ & $29.2(8.3)$ \\
\hline \multicolumn{2}{|l|}{ Protein content \% on tape strip } & II.8 (4.I) \\
\hline Layer I & $9.6(4.4)$ & $10.6(3.7)$ \\
Layer 2 & $10.1(3.3)$ & $9.0(4.0)$ \\
Layer 3 & $8.2(3.1)$ & $7.8(3.2)$ \\
Layer 4 & $8.9(2.6)$ &
\end{tabular}

Abbreviations: AU, arbitrary unit; DMM, DermaLab ${ }^{\circledR}$ Moisture Meter; TEWL, transepidermal water loss.

able to identify differences in moisture between dry lips and normal lips. The mean SquameScan-derived protein content was not significantly different between the normal and dry lip cohorts. No AEs were observed during this study.

\section{Efficacy trial: improving dry lips}

Sixty-seven subjects were enrolled $(n=34$ in the lip cream group and $n=33$ in the untreated group; Figure 6). One subject withdrew due to an AE (RHL on lower lip); therefore, 66 subjects completed the study. The mean age of the enrolled subjects was 42.6 years (range, 19-55 years).

The mean change from baseline to day 8 in visual assessment of lip dryness was greater in subjects treated with the lip cream compared to the mean change from baseline in the untreated group (adjusted mean difference, -1.48 [95\% CI: $-2.24,-0.71 ; P<0.001]$; Table 3$)$. The mean change from baseline to day 3 also favored the lip cream group $(-0.48$ [95\% CI: $-1.30,0.33 ; P=0.2408]$ ), but the difference was not statistically significant. One day after discontinuing the lip cream, mean visual dryness decreased from 2.3 on day 8 to 2.2 on day 9 . Mean corneometry measurements indicated a significant increase in skin surface hydration from baseline to day 8 in the lip cream group compared to the untreated group (adjusted mean difference, 4.62 [95\% CI: 1.05, 8.19; $P<0.05]$; Table 3). Mean (SD) corneometry ratings decreased slightly from 30.6 (10.1) on day 8 to 28.6 (9.6) on day 9. The difference in TEWL between baseline and day 8 was also statistically significant for the lip cream group compared to those who were untreated (mean difference, -7.19 [95\% CI: $-11.41,-2.98 ; P<0.01]$; Table 3 ). The subjects who applied the lip cream had a numerically greater degree of change in TEWL from baseline to day $3(-2.8)$ compared to the untreated group $(-1.3)$, but the difference was not significant (adjusted mean difference: -1.52 [95\% CI: $-5.82,2.78$; 


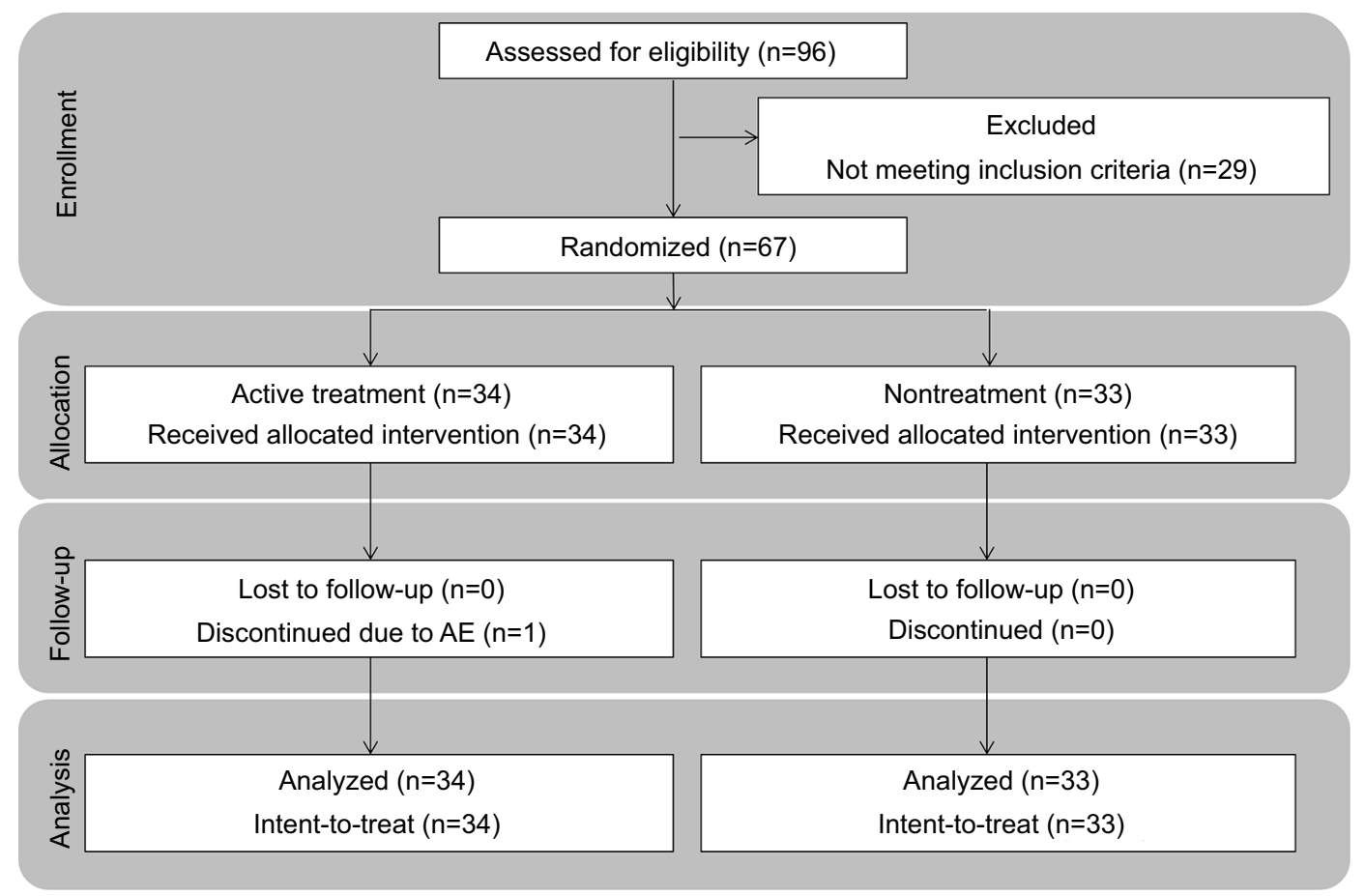

Figure 6 Study flowchart.

Abbreviation: $A E$, adverse event.

Table 3 Differences from baseline in visual assessment of dryness, corneometry, and TEWL measurements in subjects treated with lip cream vs untreated subjects

\begin{tabular}{|c|c|c|}
\hline Measurement & $\begin{array}{l}\text { Subjects treated with lip cream } \\
(n=34)\end{array}$ & $\begin{array}{l}\text { Untreated subjects } \\
(n=33)\end{array}$ \\
\hline \multicolumn{3}{|l|}{ Visual assessment of dryness ${ }^{a}$} \\
\hline Baseline & 4.7 & 4.9 \\
\hline Day 8 & 2.3 & 4.0 \\
\hline Day 9 & 2.2 & $N / A^{b}$ \\
\hline Mean change from baseline to day 8 & -2.44 & -0.97 \\
\hline Adjusted mean difference ( $P$-value) & $-1.48(P=0.0003)$ & \\
\hline \multicolumn{3}{|l|}{ Corneometer, mean (SD), F } \\
\hline Baseline & $25.7(9.4)$ & $22.7(8.5)$ \\
\hline Day 8 & $30.6(10.1)$ & $23.8(9.2)$ \\
\hline Day 9 & $28.6(9.6)$ & $N A^{b}$ \\
\hline Mean change from baseline to day 8 & 5.29 & 0.66 \\
\hline Adjusted mean difference ( $P$-value) & $4.62(P=0.0120)$ & \\
\hline \multicolumn{3}{|l|}{ TEWL, mean (SD), $g /\left(\mathbf{m}^{2} \mathbf{h}\right)$} \\
\hline Baseline & $53.2(13.6)$ & $50.0(13.4)$ \\
\hline Day 8 & $4 I .4(9.6)$ & $47.3(10.7)$ \\
\hline Day 9 & $43.0(10.9)$ & $N A^{b}$ \\
\hline Mean change from baseline to day 8 & -10.64 & -3.45 \\
\hline Adjusted mean difference ( $P$-value) & $-7.19(P=0.0011)$ & \\
\hline
\end{tabular}

Notes: ${ }^{V}$ Visual dryness of lips was assessed on a 9-point scale, in which $0=$ no dryness/chapping; $I-2=$ slight but definite roughness, fine scaling; $3-4=$ moderate roughness, coarse scaling, slight cracking; 5-6= marked roughness, coarse scaling, obvious cracking; and 7-8= very marked roughness, coarse scaling, cracked progressing to fissuring. bUtreated subjects were not assessed on day 9.

Abbreviations: NA, not applicable; TEWL, transepidermal water loss.

$P=0.4837])$. Mean (SD) TEWL increased only slightly from day $8\left(41.4[9.6] \mathrm{g} /\left(\mathrm{m}^{2} \mathrm{~h}\right)\right)$ until day $9\left(43.0[10.9] \mathrm{g} /\left(\mathrm{m}^{2} \mathrm{~h}\right)\right)$. These data show that the test lip cream strengthens the skin barrier (decreased TEWL), increases and maintains lip skin hydration (increased corneometry), and reduces dry, chapped skin (decreased visual grading scores) over time. In addition, 
the results observed 1 day after discontinuing the lip cream suggest that the product provides continued dryness protection. In summary, subjects in the treatment group experienced increasing improvement in cracking, dryness, and flaking, while subjects in the untreated group reported increases in these events at the end of the 8-day treatment period.

Analysis of the expert evaluator's photographic assessments revealed that treatment with the lip cream for 7 days provided statistically significant improvements in scaling (adjusted mean difference: -0.89 [95\% CI: $-1.75,-0.03]$; $P<0.05$ ), cupping (adjusted mean difference: -1.50 [95\% CI: $-2.30,-0.70] ; P<0.001$ ), and healthy appearance (adjusted mean difference: -1.44 [95\% CI: $-2.29,-0.58] ; P<0.01$; Figure 7A) in the treated vs untreated groups. These results are consistent with the results of clinical visual assessments for dryness reported above. For the comparative grading based on overall healthy appearance using baseline and endpoint photographs, there was a directional improvement between the treated and untreated subjects in favor of the treated group. However, the difference between the two groups was not statistically significant $(P=0.5065$; Figure 7B). A significant degree of improvement from baseline to day 8 was observed in the lip cream group $(P<0.01)$ but not in the untreated group $(P=0.0881)$. On the subject-rated assessment, subjects in the lip cream group reported improvements in cracking, dryness, and flaking, while the untreated subjects experienced worsening on these parameters (Figure 8). In addition, subjects tended to agree (3.6-3.9) that the lip cream made their lips look healthy, feel protected and moisturized, and prevented damage. The results of the tape stripping analysis did not show a significant difference between groups in the protein content of lips.

Two subjects in each group experienced a total of five AEs (three in the treatment group, two in the untreated group); one AE (ie, RHL on the lower lip) was considered possibly related to study treatment and occurred in the lip cream treatment group. Of the four AEs considered unrelated to treatment, one case each of ear infection and conjunctivitis also occurred in the lip cream treatment group, and two cases of upper respiratory infection occurred in the untreated group.

\section{Discussion}

The daily-use lip cream evaluated in these studies was specifically formulated with a novel and proprietary Micro Repair technology that forms a similar structure to the SC lipid lamellar structure while residing on the skin's surface, which together with glycerol and glucose act as barrier-protecting, barrier-enhancing, and skin-hydrating agents. ${ }^{40-47}$ In addition, UV filters are included to address the need to limit UV radiation exposure of the lips. ${ }^{1-3,48}$ Mineral oil-derived saturated hydrocarbons are not included in the formulation consistent with the request by Cosmetics Europe that their
A

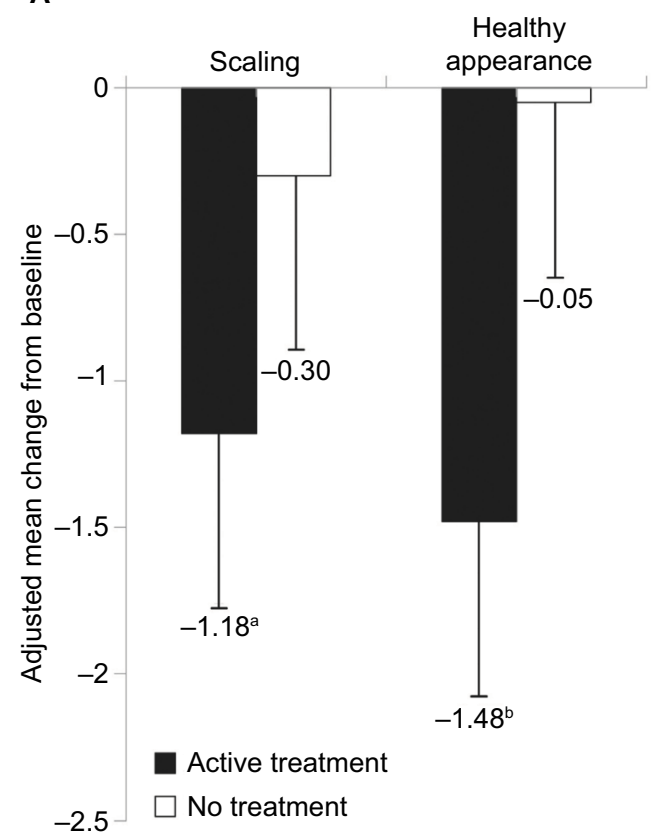

B

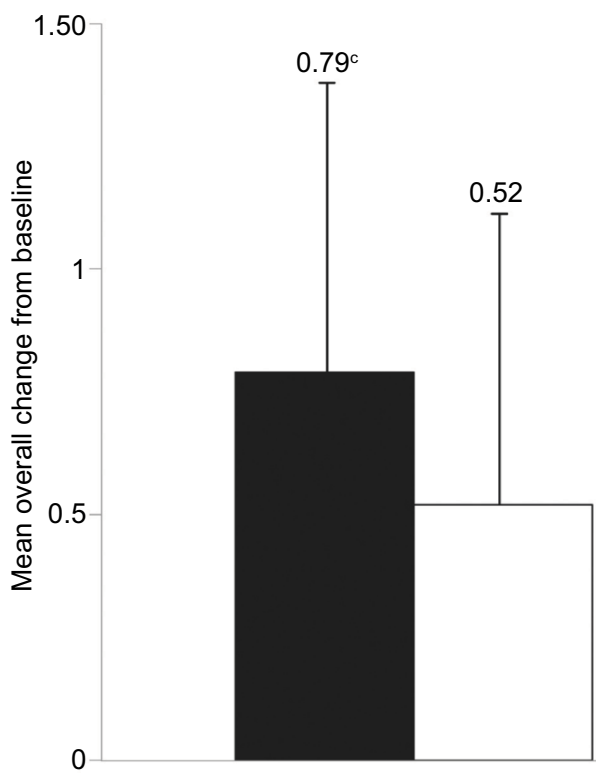

Figure 7 Composite summary of expert photographic assessments of dry lips: scaling and healthy appearance (A) and comparative grading of overall healthy appearance (B). Note: ${ }^{p}=0.04 ; \stackrel{\circ}{P}=0.001 ; \subset P=0.5065$. 
A

$\square$ 5-extremely $\square$ 4-very $\square$ 3-somewhat $\square$ 2-hardly at all $\square$ 1-not at all

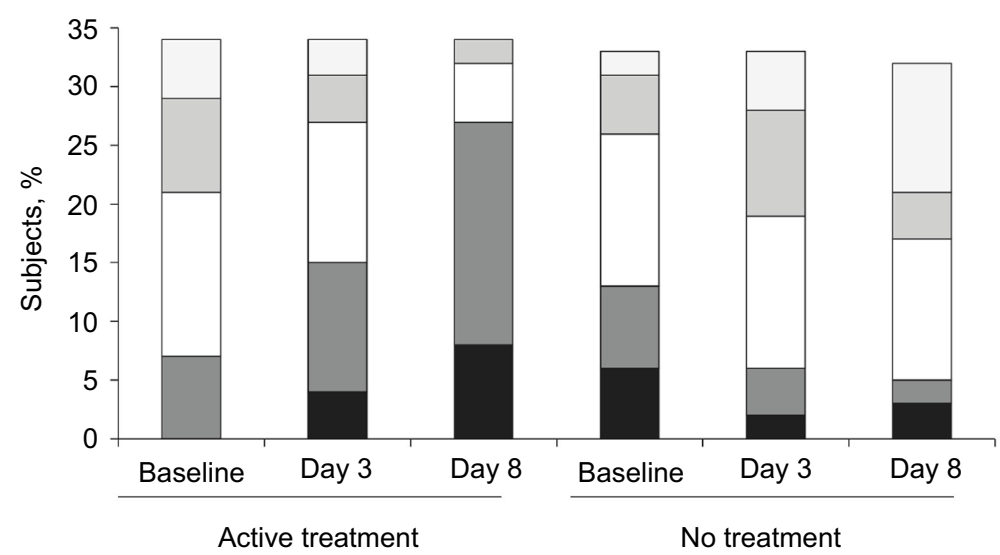

B

$\square$ 5-extremely $\square$ 4-very $\square$ 3-somewhat $\square$ 2-hardly at all $\square$ 1-not at all

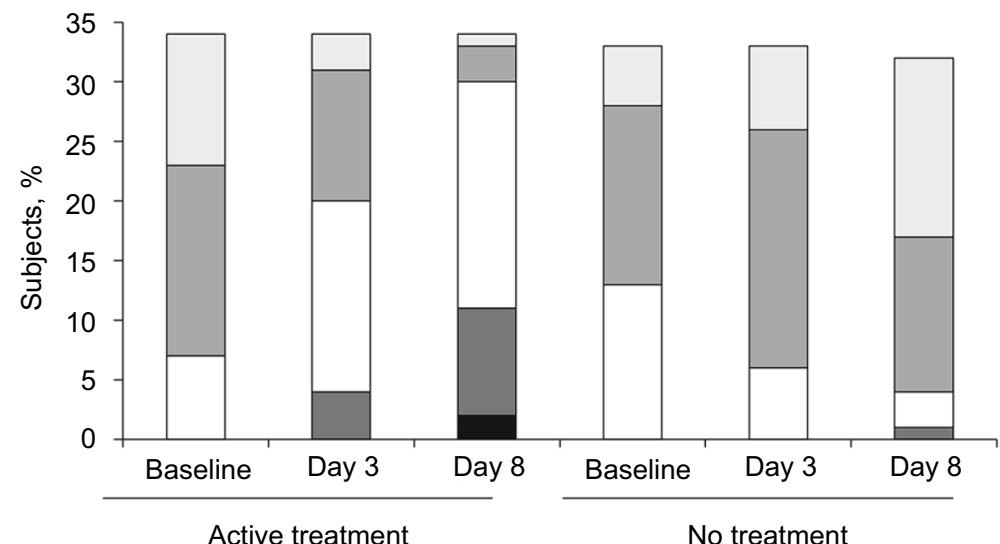

C

$\square$ 5-extremely $\square$ 4-very $\square$ 3-somewhat $\square$ 2-hardly at all $\square$ 1-not at all

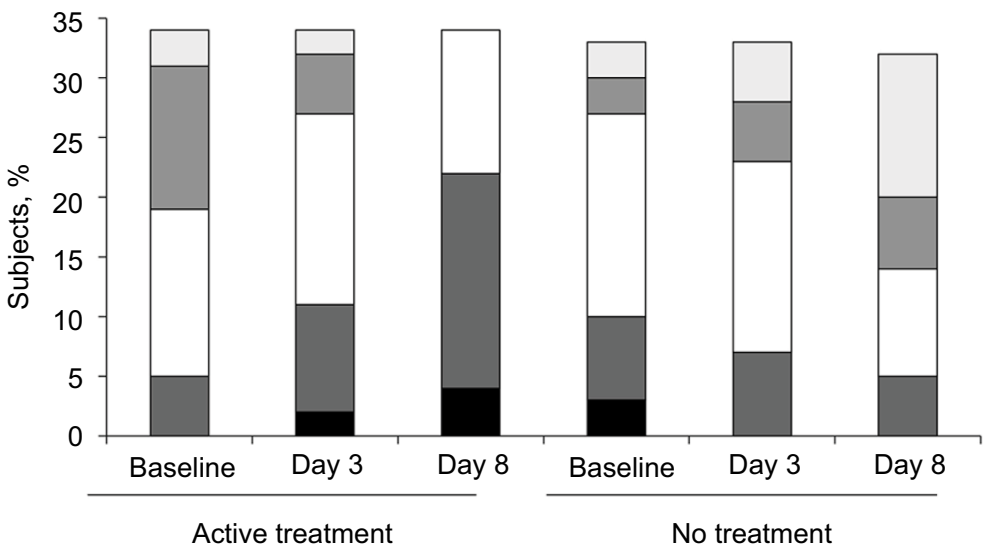

Figure 8 Summary of self-assessed physical features at baseline, day 3, and day 8: subjects with cracked lips (A), dry lips (B), and flaky lips (C). 
use is minimized in such products. ${ }^{42}$ The in vitro, in vivo, and clinical studies reported here describe the assessment of this novel daily-use lip cream for its protective activity against known environmental triggers (sun exposure and harsh winter weather conditions) of RHL and provide evidence for the effectiveness of this product against these triggers. ${ }^{19,29-32}$

Maintenance of skin hydration and barrier function is key in protecting against dehydrating weather conditions. The in vitro occlusivity study demonstrated that the inclusion of a structured hydrogenated phospholipid/behenyl alcohol/plant-derived lipid-containing emulsion provided significant occlusive properties. These values are within the range observed in our unpublished internal data with various commercial moisturizers as well as within the range of published phospholipid-structured emulsions (61.3-110.9 g/ $\left.\left(\mathrm{m}^{2} \mathrm{~h}\right)\right) .{ }^{39}$ Adequate occlusivity of a topical skin formulation is an important prerequisite to achieve meaningful in vivo skin barrier function improvement.

Minimizing UV radiation exposure is important to protect against RHL and the lip cream product demonstrated photoprotective properties in a range of experimental models. ${ }^{29-32}$ The in vitro studies demonstrated that the lip cream with sun filters reduced UV-induced inflammatory mediators as well as prevented UV-induced DNA damage (cyclobutene dimers by UVB and $80 \mathrm{OHd}$ by UVA) and apoptosis (caspase-3). These results were achieved in three distinct in vitro models: EpiDerm, EpiDerm ${ }^{\mathrm{FT}}$, and EpiGingival. Among these three models, EpiGingival tissues most closely resemble lip tissue because they consist of normal human-derived oral epithelial cells, which form the multilayered, highly differentiated models of gingival phenotypes. Our studies showed that EpiGingival tissues appeared more sensitive to UVA exposure compared to EpiDerm ${ }^{\mathrm{FT}}$ tissues. The lip cream product protected the EpiGingival tissue integrity after exposure to UVA irradiation compared to the placebo-treated tissues, further indicating the photoprotective activity of the lip cream. Moreover, these results need to be confirmed with different testing approaches such as cytometry-based apoptosis detection.

As mentioned earlier, $\mathrm{PGE}_{2}$ is an established biomarker for inflammation and is associated with UV-induced immunosuppression. ${ }^{58} \mathrm{PGE}_{2}$ has also been shown to be involved in HSV reactivation, and administration of cyclooxygenase inhibitors such as ibuprofen, indomethacin, and celecoxib has been associated with preventing reactivation of HSV. ${ }^{24-26}$ The current in vitro study demonstrates that the test lip cream reduced UV-induced $\mathrm{PGE}_{2}$ secretion and thereby may prevent RHL via this mechanism. Moreover, it has been shown that
IL-25 enhances HSV-1 replication by inhibiting filaggrin expression a mechanism that may also contribute to RHL. ${ }^{59}$ In this respect, TNF- $\alpha$, PGE $_{2}$, and IL- 6 are known to decrease filaggrin expression and their elevation by UV radiation is mitigated to some extent by the product. ${ }^{60,61}$ Although these results provide some interesting insights, more research is necessary to explore these potential mechanisms in greater detail.

In vivo the lip protection product was found to have an SPF value of 12.2 and a UVB/UVAPF of 0.9 . Only standard SPF testing was conducted on the skin of subjects' backs, which can be criticized for its anatomical relevance for a lip product. However, current SPF testing methods require large areas of the skin that preclude use of the lip as a testing site. ${ }^{54}$ Equally, UV irradiation of the lips may induce herpes lesions. ${ }^{20}$

To assess the effect of our novel lip formulation against the impact of harsh winter weather conditions on lip skin as a trigger for RHL, we first needed a study characterizing dry and normal lips to inform us about an appropriate methodology to design the in vivo efficacy study. Differences in lip hydration were apparent but unexpectedly, no statistical differences in mean TEWL measurements between subjects with dry lips and those with normal lips were observed. It was assumed that the level of dryness in recruited subjects with dry lips (grade 3 or 4 on a 10-point visual dryness assessment scale), therefore, was too low to show statistical difference in TEWL measurements compared to normal lips. Consistent with this there were no differences in SC cohesion as judged by protein measurements. Thus, for the in vivo efficacy study, the level of dryness in recruited subjects with dry lips was increased (grade 4-6 on a 9-point visual dryness assessment scale).

To the best of our knowledge, this is the first report of its kind that demonstrates improvements in the signs of lip dryness with the use of a novel sunscreen-, humectant-, and barrier lipid-containing lip cream compared to an untreated control group over the same time period. Our results show that, compared to nontreatment, 7 days of treatment with the lip cream significantly improved the visual appearance, barrier function, and moisture content of moderately dry lips. Also these benefits were maintained 24 hours after discontinuing use of the product. We found that lip condition had significantly improved in the no treatment group compared to baseline, which was most likely due to the changing weather conditions throughout the study. Subjects using the lip cream treatment perceived the product to be efficacious, as noted in the decreasing level of self-perceived flakiness, dryness, and 
cracking of their lips. In addition, the majority of subjects felt that their lips were protected and moisturized with continued use and that the product helped to prevent lip damage and strengthened their lips. Furthermore, these objective and self-assessed measurements of lip dryness were consistent with those of the photographic analysis.

The results from this series of studies provide evidence that the test lip cream formulation protects the lips against dehydration, improves SC barrier function, and protects against UVA and UVB radiation, all of which are important factors in protection against the development of RHL. ${ }^{16,18-20}$

Relatively few published studies have evaluated the efficacy of topical products for protecting the lips from stimuli known to trigger RHL. ${ }^{27,28}$ However, until recently, the use of topical sunscreen for preventing RHL had yet to be confirmed in real-life conditions. The older literature was questioned on UV protection chapping as the studies were observational in nature ${ }^{35}$ or skin was exposed to UV in a controlled environment ${ }^{29,30}$ or this observation was not replicated in subjects who applied sunscreen to their lips before and during a day of skiing. ${ }^{31}$ However, the most recent research has provided evidence for the benefit of sunscreens in preventing RHL from a randomized crossover study of a lipstick with and without sunscreen protection while subjects performed their normal daily activities including trips to the beach. ${ }^{32}$ Regarding the relationship between RHL and other weather conditions, it was shown that the harsh weather conditions of winter and having chapped lips in dry climates were significantly associated with RHL. ${ }^{19,35}$ Moreover, recently the efficacy of CS20, a protective barrier gel containing oxygenated glycerol triesters, was demonstrated in $\mathrm{RHL}^{34}$ supporting the older literature that use of lip protectants was associated with a lower prevalence of cold sore outbreaks. ${ }^{62}$

\section{Conclusion}

The daily-use lip cream formulation that was evaluated in this series of studies was specifically formulated to address the environmental triggers of RHL. It improves the skin's barrier functionality, increases and maintains the moisture content of the skin, and protects the skin against UV radiation. Additional clinical studies are necessary to establish the extent of in vivo efficacy in preventing or decreasing RHL outbreaks in individuals who are prone to RHL.

\section{Acknowledgments}

The authors wish to thank all of the subjects for their participation and Robert Geske for providing histology support.
The authors thank the proDERM Institute for Applied Dermatological Research, Schenefeld/Hamburg, Germany who conducted the in vivo SPF study between January 6 and February 5, 2014. This study was sponsored by GSK Consumer Healthcare. Medical writing assistance was provided by Dennis Stancavish, MA of Peloton Advantage and was funded by GSK Consumer Healthcare. GSK Consumer Healthcare provided a full review of the article.

\section{Disclosure}

CFG was an employee of GSK Consumer Healthcare, Weybridge, UK at the time of study planning and execution. DJM is an employee of GSK Consumer Healthcare, Weybridge, UK. HM and GS are employees of GSK Consumer Healthcare, Warren, NJ, USA. XW is an employee of GSK Consumer Healthcare, Collegeville, PA, USA. AVR is an employee of AVR Consulting, Ltd., Cheshire, UK. CBL was an employee of GSK Consumer Healthcare, Collegeville, PA, USA at the time of study planning and execution. RW is an employee of MedClin Consulting, LLC, Kolkata, India and was an employee of GSK Consumer Healthcare, Warren, NJ, USA at the time of study planning and execution. GG is an employee of cyberDERM, Inc., Broomall, PA, USA.

\section{References}

1. Kobayashi H, Tagami H. Functional properties of the surface of the vermilion border of the lips are distinct from those of the facial skin. Br J Dermatol. 2004;150(3):563-5671.

2. Zugerman C. The lips: anatomy and differential diagnosis. Cutis. 1986;38(2):116-120.

3. Gabard B, Ademola J. Lip sun protection factor of a lipstick sunscreen. Dermatology. 2001;203(3):244-247.

4. Brenner M, Hearing VJ. The protective role of melanin against UV damage in human skin. Photochem Photobiol. 2008;84(3):539-549.

5. Ya-Xian Z, Suetake T, Tagami H. Number of cell layers of the stratum corneum in normal skin - relationship to the anatomical location on the body, age, sex and physical parameters. Arch Dermatol Res. 1999;291(10):555-559.

6. Ishikawa J, Shimotoyodome Y, Ito S, et al. Variations in the ceramide profile in different seasons and regions of the body contribute to stratum corneum functions. Arch Dermatol Res. 2013;305(2):151-162.

7. Yang Q, Guy RH. Characterisation of skin barrier function using bioengineering and biophysical techniques. Pharm Res. 2015;32(2):445-457.

8. Tamura E, Ishikawa J, Naoe A, Yamamoto T. The roughness of lip skin is related to the ceramide profile in the stratum corneum. Int J Cosmet Sci. 2016;38(6):615-621.

9. Looker KJ, Magaret AS, May MT, et al. Global and regional estimates of prevalent and incident herpes simplex virus type 1 infections in 2012 PLoS One. 2015;10(10): 0140765.

10. Embil JA, Stephens RG, Manuel FR. Prevalence of recurrent herpes labialis and aphthous ulcers among young adults on six continents. Can Med Assoc J. 1975;113(7):627-630.

11. Young SK, Rowe NH, Buchanan RA. A clinical study for the control of facial mucocutaneous herpes virus infections. I. Characterization of natural history in a professional school population. Oral Surg Oral Med Oral Pathol. 1976;41(4):498-507. 
12. Esmann J. The many challenges of facial herpes simplex virus infection. J Antimicrob Chemother. 2001;47(Suppl T1):17-27.

13. Perna JJ, Mannix ML, Rooney JF, Notkins AL, Straus SE. Reactivation of latent herpes simplex virus infection by ultraviolet light: a human model. J Am Acad Dermatol. 1987;17(3):473-478.

14. Blyth WA, Hill TJ, Field HJ, Harbour DA. Reactivation of herpes simplex virus infection by ultraviolet light and possible involvement of prostaglandins. J Gen Virol. 1976;33(3):547-550.

15. Harbour DA, Hill TJ, Blyth WA. Recurrent herpes simplex in the mouse: inflammation in the skin and activation of virus in the ganglia following peripheral stimulation. J Gen Virol. 1983;64(Pt 7):1491-1498.

16. Hill TJ, Blyth WA, Harbour DA. Trauma to the skin causes recurrence of herpes simplex in the mouse. J Gen Virol. 1978;39(1):21-28.

17. Chida Y, Mao X. Does psychosocial stress predict symptomatic herpes simplex virus recurrence? A meta-analytic investigation on prospective studies. Brain Behav Immun. 2009;23(7):917-925.

18. Taylor JR, Schmieder GJ, Shimizu T, Tie C, Streilein JW. Interrelationship between ultraviolet light and recurrent herpes simplex infections in man. J Dermatol Sci. 1994;8(3):224-232.

19. Berg J, Kao C, Browne J, Barrett K. Characterization of a herpes simplex labialis population. J Am Acad Dermatol. 2009;60(3 Suppl 1):AB35.

20. Sayre RM, Dowdy JC, Harris KA, Berg JE, Trimble MW. A practical UV source to induce Herpes simplex labialis lesions in the clinic. Photoderm Photoimm Photomed. 2007;23(1):20-23.

21. Fisher GJ, Wang Z, Datta SC, Varani J, Kang S, Voorhees JJ. Pathophysiology of premature skin aging induced by ultraviolet light. $N$ Engl J Med. 1997;337(20):1419-1429.

22. Clydesdale GJ, Dandie GW, Muller HK. Ultraviolet light induced injury: immunological and inflammatory effects. Immunol Cell Biol. 2001;79(6):547-568.

23. Sander WJ, O'Neill HG, Pohl CH. Prostaglandin E2 as a modulator of viral infections. Front Physiol. 2017;8:89.

24. Gebhardt BM, Varnell ED, Kaufman HE. Inhibition of cyclooxygenase 2 synthesis suppresses Herpes simplex virus type 1 reactivation. J Ocul Pharmacol Ther. 2005;21(2):114-120.

25. Harbour DA, Blyth WA, Hill TJ. Prostaglandins enhance spread of herpes simplex virus in cell cultures. J Gen Virol. 1978;41(1):87-95.

26. Wachsman M, Aurelian L, Burnett JW. The prophylactic use of cyclooxygenase inhibitors in recurrent herpes simplex infections. $\mathrm{Br} J$ Dermatol. 1990;123(3):375-380.

27. Chon T, Nguyen L, Elliott TC. Clinical inquiries. What are the best treatments for herpes labialis? J Fam Pract. 2007;56(7):576-578.

28. Opstelten W, Neven AK, Eekhof J. Treatment and prevention of herpes labialis. Can Fam Physician. 2008;54(12):1683-1687.

29. Duteil L, Queille-Roussel C, Loesche C, Verschoore M. Assessment of the effect of a sunblock stick in the prevention of solar-simulating ultraviolet light-induced herpes labialis. J Dermatolog Treat. 1998;9(1): $11-14$.

30. Rooney JF, Mannix ML, Wohlenberg CR, et al. Prevention of ultraviolet-light-induced herpes labialis by sunscreen. Lancet. 1991;338(8780):1419-1422.

31. Mills J, Hauer L, Gottlieb A, Dromgoole S, Spruance S. Recurrent herpes labialis in skiers. Clinical observations and effect of sunscreen. Am J Sports Med. 1987;15(1):76-78.

32. Mazzarello V, Ferrari M, Piu G, Pomponi V, Solinas G. Do sunscreen prevent recurrent Herpes labialis in summer? J Dermatolog Treat. 2018;38:1-4.

33. Godfrey HR, Godfrey NJ, Godfrey JC, Riley D. A randomized clinical trial on the treatment of oral herpes with topical zinc oxide/glycine. Altern Ther Health Med. 2001;7(3):49-56.

34. Khemis A, Duteil L, Coudert AC, Tillet Y, Dereure O, Ortonne JP. Evaluation of the efficacy and safety of a CS20® protective barrier gel containing OGT compared with topical aciclovir and placebo on functional and objective symptoms of labial herpes recurrences: a randomized clinical trial. J Eur Acad Dermatol Venereol. 2012;26(10):1240-1246.

35. Shulman JD, Carpenter WM, Lewis DL. The prevalence of recurrent herpes labialis during an army hot weather exercise. J Public Health Dent. 1992;52(4):198-203.
36. Trookman NS, Rizer RL, Ford R, Mehta R, Gotz V. Clinical assessment of a combination lip treatment to restore moisturization and fullness. J Clin Aesthet Dermatol. 2009;2(12):44-48.

37. Lopez-Jornet P, Camacho-Alonso F, Rodriguez-Espin A. Study of lip hydration with application of photoprotective lipstick: influence of skin phototype, size of lips, age, sex and smoking habits. Med Oral. 2009;15(3):e445-e450.

38. Isoda K, Nakamura T, Yoshida K, et al. The efficacy of a lip balm containing pseudo-ceramide on the dry lips of sensitive skin-conscious subjects. J Cosmet Dermatol. 2018;17(1):84-89.

39. Bulsara PA, Varlashkin P, Dickens J, Moore DJ, Rawlings AV, Clarke MJ. The rational design of biomimetic skin barrier lipid formulations using biophysical methods. Int J Cosmet Sci. 2017;39(2):206-216.

40. Gooris GS, Kamran M, Kros A, Moore DJ, Bouwstra JA. Interactions of dipalmitoylphosphatidylcholine with ceramide-based mixtures. Biochim Biophys Acta Biomembr. 2018;1860(6):1272-1281.

41. Ghadially R, Halkier-Sorensen L, Elias PM. Effects of petrolatum on stratum corneum structure and function. J Am Acad Dermatol. 1992;26(3):387-396.

42. Niederer M, Stebler T, Grob K. Mineral oil and synthetic hydrocarbons in cosmetic lip products. Int J Cosmet Sci. 2016;38(2):194-200.

43. Food and Drug Administration. HHS. Skin protectant drug products for over-the-counter human use; final monograph. Final rule. Fed Regist. 2003;68(107):33362-33381.

44. Szél E, Polyánka H, Szabó K, et al. Anti-irritant and anti-inflammatory effects of glycerol and xylitol in sodium lauryl sulphate-induced acute irritation. J Eur Acad Dermatol Venereol. 2015;29(12): 2333-2341.

45. Korponyai C, Szél E, Behány Z, et al. Effects of locally applied glycerol and xylitol on the hydration, barrier function and morphological parameters of the skin. Acta Derm Venerol. 2017;97(2): 182-187.

46. Yamada K, Matsushita K, Wang J, Kanekura T. Topical glucose induces claudin-1 and filaggrin expression in a mouse model of atopic dermatitis and in keratinocyte culture, exerting anti-inflammatory effects by repairing skin barrier function. Acta Derm Venerol. 2018;98(1):19-25.

47. Páyer E, Szabó-Papp J, Ambrus L, et al. Beyond the physico-chemical barrier: glycerol and xylitol markedly yet differentially alter gene expression profiles and modify signalling pathways in human epidermal keratinocytes. Exp Dermatol. 2018;27(3):280-284.

48. Olsen CM, Wilson LF, Green AC, Biswas N, Loyalka J, Whiteman DC. Prevention of DNA damage in human skin by topical sunscreens. Photodermatol Photoimmunol Photomed. 2017;33(3):135-142.

49. Afaq F, Zaid MA, Khan N, Dreher M, Mukhtar H. Protective effect of pomegranate-derived products on UVB-mediated damage in human reconstituted skin. Exp Dermatol. 2009;18(6):553-561.

50. Pygmalion MJ, Ruiz L, Popovic E, et al. Skin cell protection against UVA by Sideroxyl, a new antioxidant complementary to sunscreens. Free Radic Biol Med. 2010;49(11):1629-1637.

51. Agrawal A, Shindell E, Jordan F, Baeva L, Pfefer J, Godar DE. UV radiation increases carcinogenic risks for oral tissues compared to skin. Photochem Photobiol. 2013;89(5):1193-1198.

52. Marionnet C, Bernerd F. Organotypic models for evaluating sunscreens. In: Wang S, Lim H, editors. Principles and Practice of Photoprotection. Switzerland: Springer International, Adis; 2016:199-225.

53. Questions and answers: FDA announces new requirements for over-thecounter (OTC) sunscreen products marketed in the U.S; 2011. Available from: http://www.fda.gov/Drugs/ResourcesForYou/Consumers/BuyingUsingMedicineSafely/UnderstandingOver-the-CounterMedicines/ ucm258468.htm. Accessed December 21, 2016.

54. Cosmetics - Sun Protection Test Methods - In Vivo Determination of the Sun Protection Factor (SPF) (ISO 24444:2010). London: British Standard; 2010.

55. Santos Caetano JP, Abarca AP, Guerato M, et al. SPF and UVA-PF sunscreen evaluation: are there good correlations among results obtained in vivo, in vitro and in a theoretical Sunscreen Simulator? A real-life exercise. Int J Cosmet Sci. 2016;38(6):576-580. 
56. The Commission of the European Communities. Commission recommendation of 22 September 2006 on the efficacy of sunscreen products and the claims made relating thereto. Off $J$ Eur Union. 2006;L265:39-43.

57. Voegeli R, Heiland J, Doppler S, Rawlings AV, Schreier T. Efficient and simple quantification of stratum corneum proteins on tape strippings by infrared densitometry. Skin Res Technol. 2007;13(3):242-251.

58. Prasad R, Katiyar SK. Prostaglandin E2 promotes UV radiation-induced immune suppression through DNA hypermethylation. Neoplasia. 2013;15(7):795-804.

59. Kim BE, Bin L, Ye YM, Ramamoorthy P, Leung DYM. IL-25 enhances HSV-1 replication by inhibiting filaggrin expression, and acts synergistically with Th2 cytokines to enhance HSV-1 replication. J Invest Dermatol. 2013;133(12):2678-2685.
60. Lee CW, Lin ZC, Hu SC, et al. Urban particulate matter down-regulates filaggrin via COX2 expression/PGE2 production leading to skin barrier dysfunction. Sci Rep. 2016;6(1):27995.

61. Kim BE, Howell MD, Guttman-Yassky E, et al. TNF- $\alpha$ downregulates filaggrin and loricrin through c-Jun $\mathrm{N}$-terminal kinase: role for TNF- $\alpha$ antagonists to improve skin barrier. J Invest Dermatol. 2011;131(6):1272-1279.

62. Shulman JD, Lewis DL, Carpenter WM. The prevalence of chapped lips during an army hot weather exercise. Mil Med. 1997;162(12):817-819. 


\section{Supplementary materials}

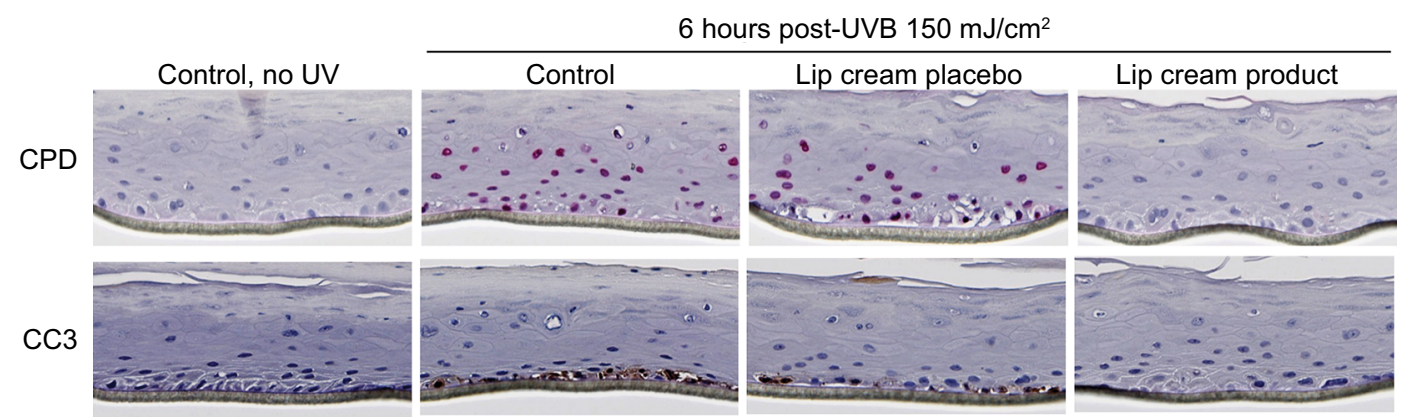

Figure SI Lip cream with UV filters inhibited UVB DNA damage (CPD, pink staining) and apoptosis (CC3, brown staining) in EpiGingival ${ }^{\text {TM }}$.

Notes: Lip cream with UV filters and without UV filters (placebo) were topically applied I hour prior to UVB irradiation (I50 mJ/cm²). Non-UVB treatment (untreated) was used as a negative control and baseline. Tissue samples were collected for IHC staining at 6 hours post-UVB $(n=3)$. The images at top panels were scanned at $40 \times$ using Nanozoomer (Hamamatsu) and shown at 100\%.

Abbreviations: CC3, cleaved caspase-3; CPD, cyclobutane pyrimidine dimers; IHC, immunohistochemical; UVB, ultraviolet B.

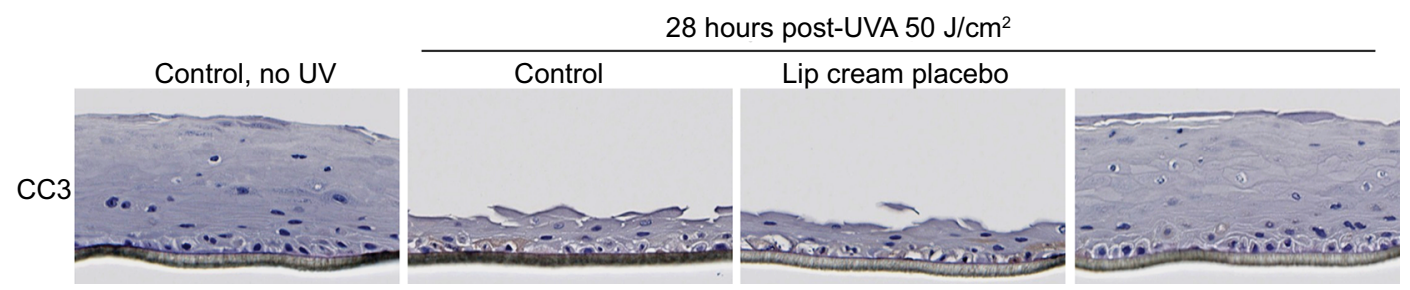

Figure S2 Lip cream with UV filters inhibited UVA-induced apoptosis (CC3, brown staining) in EpiGingival ${ }^{\mathrm{TM}}$ at 28 hours post-UVA (50 J/cm²) after UVA irradiation. Notes: Lip cream placebo is the test formulation without the UV filters. Untreated samples were not applied with any test product ( $\mathrm{n}=3$ ).

Abbreviations: CC3, cleaved caspase-3; UVA, ultraviolet A.

\section{Publish your work in this journal}

Clinical, Cosmetic and Investigational Dermatology is an international, peer-reviewed, open access, online journal that focuses on the latest clinical and experimental research in all aspects of skin disease and cosmetic interventions. This journal is included on PubMed. The manuscript management system is completely online and includes a very quick and fair peer-review system, which is all easy to use. Visit http://www.dovepress.com/testimonials.php to read real quotes from published authors 